\title{
TEXTOS ESCRITOS EN ESPAÑOL COMO SEGUNDA LENGUA POR ESTUDIANTES SORDOS BILINGÜES: UN ANÁLISIS DESDE LA LINGÜÍSTICA SISTÉMICO-FUNCIONAL
}

\author{
Texts Written in Spanish as a Second Language by Deaf Bilingual Students: \\ A Systemic-Functional Linguistics Analysis
}

TEXTES ÉCRITS EN ESPAgNOL COMME LANGUE SECONDE PAR DES ÉTUDIANTS SOURDS BILINGUES : UNE ANALYSE BASÉE SUR LA LINGUISTIQUE SYSTÉMIQUE-FONCTIONNELLE

\author{
TEXTOS ESCRITOS EM ESPANHOL COMO SEgUNDA LÍNGUA POR ESTUdANTES SURdos \\ BILÍNGÜES: UMA ANÁLISE BASEADA NA LINGUÍSTICA SISTÊMICO-FUNCIONAL
}

\author{
Diana Lucía Valencia-Méndez \\ Docente, Institución Universitaria \\ Escuela Nacional del Deporte, Cali, \\ Colombia. \\ dianaluciavalencia@gmail.com \\ http://orcid. \\ org/0000-0002-9483-5505
}

Este texto surge como producto de la investigación "La metafunción ideacional lógica en los textos escritos en español por estudiantes Sordos bilingües de bachillerato", llevada a cabo entre febrero de 2017 y abril de 2018, en el marco de la Maestría en Estudios Interlingüísticos e Interculturales de la Universidad del Valle

\begin{abstract}
RESUMEN
La escritura en español por estudiantes Sordos ha sido valorada, frecuentemente, con base en las producciones de estudiantes oyentes monolingües coetáneos, ignorando que se trata de un proceso escrito bilingüe bicultural. Este artículo presenta una descripción del español escrito como segunda lengua por cuatro estudiantes Sordos bilingües de último año de secundaria de la ciudad de Cali, usuarios de la lengua de señas colombiana. Se realizó un estudio descriptivo en el que se incluyeron entrevistas a los estudiantes y se recolectaron textos escritos. Estos datos permitieron analizar tanto la historia sociolingüística de los estudiantes como las funciones que cumplen las diferentes palabras. Para lograrlo, se utilizó la clasificación de Halliday en la lingüística sistémico-funcional: grupos, frases y clases de palabras. Se encontró adquisición tardía de ambas lenguas y escritura en español no estandarizado, con alta frecuencia de diversos grupos nominales, grupos verbales y frases preposicionales, y con baja frecuencia de grupos adverbiales, grupos conjuntivos y grupos preposicionales. Se advirtió que algunas de las variaciones lingüísticas podrían asociarse a la interlengua y a transferencias con la lengua de señas colombiana, lo cual es similar a lo que sucede con aprendices bilingües oyentes, con la diferencia de que la comunidad Sorda es una minoría usuaria de lenguas con diferentes modalidades y estatus social. También se observó que hay ausencia de expertos en educación bilingüe bicultural.

Palabras clave: estudiantes sordos; educación bilingüe; escritura en español; español como segunda lengua; lengua de señas colombiana; lingüística sistémico-funcional.
\end{abstract}

Recibido: 2021-01-20 / Aceptado: 2021-06-11 / Publicado: 2022-02-11

https://doi.org/10.17533/udea.ikala.v27n1a01

Editora: Doris Correa, Universidad de Antioquia, Medellín, Colombia.

Derechos patrimoniales, Universidad de Antioquia, 2022. Este es un artículo en acceso abierto, distribuido según los términos de la licencia Creative Commons BY-NC-SA 4.0 Internacional. 


\section{Abstract}

Spanish language writing by Hearing-impaired students has often been judged on the same scale as peer monolingual hearing students' productions, overlooking the fact that it involves a bicultural bilingual writing process. This article provides a description of written Spanish as a second language by four bilingual hearingimpaired students, who are themselves Colombian sign language users in their last year of high school in the city of Cali, Colombia. The descriptive study involved interviews with the students and a collection of their written texts. This input allowed us to analyse both the students' sociolinguistic background and the roles performed by different words. In order to do that, we turned to Halliday's systemic-functional linguistics classification of words in groups, phrases and categories. Findings showed late acquisition of both languages and writing in non-standard Spanish, with highly frequent occurrence of varied noun phrases, verbal groups and prepositional phrases; and low frequency use of adverb groups, conjunction groups, and prepositional groups. It was noted that some linguistic variations might be related to an interlanguage and to a transference from Colombian sign language, which is similar to what happens with hearing bilingual learners, with the difference that the Deaf community is a minority in language use, with different modalities and social status. Finally, findings included a reported absence of experts in bicultural bilingual education.

Keywords: hearing-impaired students; bilingual education; writing in Spanish; Spanish as a second language; Colombian sign language; systemic-functional linguistics.

\section{RÉSUMÉ}

L'écriture en espagnol par des étudiants sourds a été valorisée, fréquemment, sur la base des productions d'étudiants entendants monolingues du même âge, ignorant qu'il s'agit d'un processus d'écriture biculturelle bilingue. Cet article présente une description de l'espagnol écrit comme langue seconde par quatre élèves sourds bilingues en dernière année de lycée dans la ville de Cali. Les élèves utilisent la langue des signes colombienne. Une étude descriptive a été réalisée qui comprenait des entretiens avec les étudiants et une collection de textes écrits. Dans ceux-ci, nous avons analysé l'histoire sociolinguistique des étudiants ainsi que les fonctions que remplissent les différents mots. Pour ce faire, nous avons suivi la classification proposée par Halliday dans la linguistique systémiquefonctionnelle : en groupes, phrases et classes de mots. Les conclusions de l'étude incluent une acquisition tardive des deux langues et une écriture non standardisée en espagnol, avec une fréquence élevée de divers groupes nominaux, groupes verbaux et phrases prépositionnelles, et une faible fréquence de groupes adverbiaux, conjonctifs et prépositionnels. Aussi certaines variations linguistiques pourraient être associées à l'interlangue et aux transferts avec la langue des signes colombienne, ce qui est similaire à ce qui se passe avec les apprenants bilingues entendants, à la différence que la communauté sourde est un utilisateur minoritaire de langues avec des modalités et statut social divers. Finalement nous avons observé une absence d'experts en éducation bilingue biculturelle.

Mots-clefs : étudiants sourds; enseignement bilingue ; écriture en espagnol; espagnol langue seconde; langue des signes colombienne; linguistique systémique-fonctionnelle. 


\section{Resumo}

A escrita em espanhol por alunos surdos tem sido valorizada, frequentemente, a partir das produções de alunos monolíngues contemporâneos, ignorando que se trata de um processo de escrita bilíngue bicultural. Este artigo apresenta uma descrição do espanhol escrito como segunda língua por quatro alunos surdos bilíngues do último ano do ensino médio da cidade de Cali, usuários da língua de sinais colombiana. Realizou-se um estudo descritivo que incluiu entrevistas com alunos e coleta de textos escritos. Nestes, foi analisada a história sociolinguística dos alunos e as funções que as diferentes palavras desempenham. Com esse fim, utilizou-se a classificação proposta por Halliday na linguística sistêmico-funcional: em grupos, frases e classes de palavras. Os resultados indicam aquisição tardia de ambas as línguas e escrita em espanhol não padronizado, com grande frequência de vários grupos nominais, grupos verbais e frases preposicionais, e menor frequência de grupos adverbiais, conjuntivos e proposicionais. Observou-se que algumas das variações linguísticas podem estar associadas à interlíngua e transferências com a língua de sinais colombiana, o que é semelhante ao que acontece com alunos bilíngues ouvintes, com a diferença de que a comunidade Surda é uma minoria usuária de línguas com diferentes. modalidades e status social. Ainda é notória a ausência de especialistas em educação bilíngue bicultural.

Palavras chave: estudiantes surdos; educação bilíngue; escrita em espanhol; espanhol como segunda língua; língua de sinais colombiana; linguística sistêmico-funcional. 


\section{Introducción}

La concepción del Sordo desde la perspectiva socioantropológica ha generado, desde los años noventa, en Colombia, una propuesta educativa bilingüe bicultural, que promueve la lengua de señas colombiana (LSC) como primera lengua (L1) y el español como segunda lengua (L2) (Carvajal, 2009). Actualmente, los estudiantes Sordos se integran en instituciones de oyentes y comparten sus clases con ellos, mientras reciben el acompañamiento de un intérprete de LSC.

Desde esta perspectiva, se escribe "Sordo" con mayúscula para denominar a personas que no oyen y han desarrollado una identidad y una cultura fundamentada en el aprendizaje visual y la lengua de señas (LS), diferenciándose del concepto de sordo con minúscula, correspondiente a una visión clínica de la hipoacusia (Massone y Machado, 1994; Muñoz, 2020).

16 Aunque se han hecho esfuerzos por la inclusión y el uso de ambas lenguas en el contexto educativo, la comunidad Sorda sigue teniendo dificultades para alcanzar niveles avanzados de proficiencia en estas y de escolaridad. En el año 2018, solo el $8 \%$ de 52234 personas sordas entre los 6 y 18 años estaba matriculada en Colombia y al menos el $80 \%$ de quienes presentaron la prueba SABER fueron clasificados en el nivel de desempeño más bajo en las pruebas de lectura crítica (Colombia, Ministerio de Educación Nacional e Instituto Nacional para Sordos, 2019). Situaciones semejantes también se presentan en países desarrollados como Estados Unidos, donde solo el $27 \%$ de los estudiantes de grado $12 .^{\circ}$ tiene alta competencia en la escritura (Mayer y Trezek, 2019). En cualquier caso, esto se constituye en un problema para garantizar el desempeño de las personas Sordas en la educación superior y en la vida laboral (Scott y Hoffmeister, 2018), lo que propicia la marginación en una sociedad mayoritariamente oyente (L. Tovar, 2004).

En parte, esta problemática se relaciona con que en el sector educativo hay desconocimiento de aspectos de la educación bilingüe-bimodal, como las metodologías particulares, la necesidad de personal suficientemente instruido en bilingüismo y LS, los materiales específicos de evaluación y trabajo en el aula, etc. También se reconoce que falta más investigación en áreas como la enseñanza de la escritura a personas Sordas (Mayer y Trezek, 2019; Williams y Mayer, 2015). Esto ha generado una tendencia a usar metodologías que originariamente fueron creadas para oyentes y a comparar su competencia lingüística con la de la población Sorda (Celemín, 2014; Flórez et al., 2010; Herrera, 2014; Herrera y Paz, 2019; Lissi et al., 2012; Luna, 2014; Mayer y Trezek, 2019; Morales, 2009; Pacheco y Anzola, 2011; Rusell, 2016; Scott y Hoffmeister, 2018; L. Tovar, 2002; 2005; Zambrano, 2008).

Lo anterior conduce, en muchos casos, a concebir las producciones de las personas Sordas desde el error o la deficiencia, y a desconocerlas como aprendices bilingües, que transitan entre culturas y lenguas de diferente modalidad, por lo que experimentan fenómenos propios del contacto entre lenguas, como las transferencias, las divergencias y los cambios en la sintaxis (Galvis, 2005; Herrera, 2014; K. Tovar, 2016; Lissi et al., 2012; Massone et al., 2005; Menéndez, 2012; Rusell, 2016; Scott y Hoffmeister, 2018; Zambrano, 2008).

En este sentido, este artículo describe el español escrito por estudiantes Sordos bilingües de último grado de educación secundaria. Presenta los grupos, las frases y las relaciones entre cláusulas hallados en la muestra, mediante el modelo de la lingüística sistémico-funcional de Halliday (2014), que concibe el estrato léxico-gramatical en relación con el uso de la lengua que hacen los hablantes para manifestar su pensamiento y no solo como un conjunto de reglas lingüísticas.

Además, contempla la posibilidad de que muchos de los hallazgos que no corresponden al español estándar se pueden relacionar con la hipótesis de la interlengua (Selinker, 1972), lo que permite reconocer la influencia que tiene la Ls en la adquisición 
de la L2 como parte de un proceso natural del bilingüismo, sin reducir las ocurrencias alejadas de la norma a "errores".

\section{Marco teórico}

Desde el paradigma socioantropológico, se concibe a la persona Sorda como ser diferente, no deficiente, poseedor de la LS, símbolo de identidad y de pertenencia a la comunidad Sorda, que es lingüísticamente minoritaria y heterogénea (Massone et al., 1999; Rodríguez, 2013; L. Tovar, 2004).

A continuación se presentan los conceptos de bilingüismo, interlengua y lingüistica sistémicofuncional. Por último, se exponen algunos estudios que sirven como antecedentes y bases para el análisis de la lengua escrita de las personas Sordas.

\section{Bilingüismo}

En este artículo se acoge la clasificación de bilingüismo dada por Appel y Muysken (2005). La primera es el bilingüismo social, que afirma que casi todas las sociedades son bilingües, pero el nivel de bilingüismo varía de una sociedad a otra. Así, en Colombia, las personas Sordas son una minoría bilingüe en los sentidos sociológico y estadístico, y los oyentes, que son mayoría, manejan el poder y tienden a ser monolingües.

La segunda es el bilingüismo individual, relacionado con el uso que una persona hace de las lenguas que conoce, entendiendo que el desarrollo de las habilidades lingüísticas variará de acuerdo con las funciones que dichas lenguas cumplan en los diferentes dominios de la sociedad (Romaine, 1996) y con las necesidades personales. En el caso de las personas Sordas, L. Tovar (1995) expone que la LS cumple con funciones inferiores, relacionadas con dominios restringidos al hogar, al barrio y al culto religioso, mientras que la lengua oficial, el español en este caso, se emplea para las funciones de orden superior, relacionadas con el poder y la educación.

\section{Aspectos afectivos del bilingüismo}

En la adquisición de L2, Brown (2007) plantea la importancia de reconocer la influencia de los sentimientos o emociones en los comportamientos de los aprendices de L2, ya que el bilingüismo no está mediado únicamente por variables cognitivas. El autor expone que "El desarrollo de estados afectivos o sentimientos involucra una variedad de factores de personalidad, sentimientos tanto sobre nosotros mismos como sobre los demás con los que entramos en contacto" (Brown, 2017, p. 140). Esto es relevante si se tiene en cuenta que la persona se relacionará con la nueva lengua y cultura de acuerdo con la valoración y las sensaciones que vaya experimentando en la medida en que se acerca a ellas.

El primer aspecto afectivo que se quiere considerar es la autoestima, que se relaciona con "el concepto de sí mismo de una persona, la aceptación de sí mismo y el reflejo de sí mismo como se ve en la interacción entre uno mismo y los demás" (Brown, 2007, p. 141). Esta influye en el éxito que tenga el aprendiz sobre la L2 y la forma en que percibe aspectos culturales que se asocian al proceso. $\mathrm{Al}$ respecto, los docentes cumplen un papel que puede incidir positivamente en el desempeño lingüístico y el bienestar emocional de los estudiantes.

El segundo es el deseo de comunicarse, que es la voluntad que una persona tiene para iniciar o evitar una interacción con otro. Es un factor que no depende solo de tener una alta habilidad comunicativa, sino también de otros factores emocionales y personales. El deseo de comunicarse será más alto si la persona recibe apoyo de las demás personas para comunicarse y tiene impacto sobre la concepción social que el aprendiz tiene de sí mismo. Esto se puede relacionar con la explicación de Carvajal (2009) sobre la forma en que se puede debilitar la intención de comunicación de las personas Sordas con las oyentes cuando perciben que estas solo deletrean y usan pocas señas, lo que aumenta 
la desmotivación y genera aislamiento del grupo Sordo respecto al de los oyentes.

El tercero es la empatía, relacionada con el reconocimiento del sentir ajeno, la cual es más fácil de alcanzar por medio de la comunicación oral que de la escrita; por tanto, si entre Sordos y oyentes no hay comunicación fluida en LSC, será más difícil generarla para facilitar la comprensión del otro.

El último es la motivación, que es el esfuerzo y la toma de decisiones que una persona hace por alcanzar un objetivo según sus necesidades personales y el contexto en que se desenvuelve. La motivación puede ser global, situacional u orientada a una tarea. Así, quienes están motivados a aprender a leer se esfuerzan por lograrlo, porque comprenden el valor de la lectura, pero si la consideran inútil, no lo harán. También es posible que el grado de motivación que tenga una persona Sorda de aprender la L2 a nivel global sea escrita puede ser baja, lo que influirá en el nivel de desempeño que alcance en esta.

\section{Interlengua}

La interlengua (Selinker, 1972) es una gramática provisional de la L2, generada por los aprendices bilingües mientras van avanzando en su proceso de bilingüismo. Tiene características de la primera y la segunda lengua, y va evolucionando y acercándose a la lengua meta (L2). Adicionalmente, Larsen-Freeman (2014) explica que la interlengua es cambiante, dinámica y que el usuario de la L2 no necesariamente llegará a hablar como un nativo.

La interlengua permite entender que la escritura de la comunidad Sorda puede tener características de la LS y de la L2, ambas con modalidades diferentes: una visogestual y otra escrita. Por ejemplo, en la estructura sintáctica de una cláusula del español, una persona Sorda puede omitir un grupo o parte de él, en tanto en la primera lengua este se produce mediante un rasgo no manual, mientras

que en la L2 se organizan de forma consecutiva y lineal.

Por lo anterior, se considera que al hablar de interlengua es propicio el concepto de divergencia (Menéndez, 2012), para referirse a las ocurrencias alejadas de la norma lingüística, que, en este caso, surgen por el contacto entre la LS y la L2, ya sea por causas intralingüísticas, propias del desarrollo lingüístico, o por causas interlingüísticas, como ocurre con las transferencias.

Esto permite dejar de lado la noción de "error", por considerarla inapropiada para hablar de procesos de aprendizaje bilingüe intermodal, como sucede en el caso de la comunidad Sorda.

Por su parte, Galvis (2005) expone que en fases iniciales de adquisición de la L2 hay omisión de artículos, pronombres, preposiciones, verbos, especialmente de ser y estar. Y Ruiz (2011) concluye que la falta de concordancia entre sujeto y verbo provoca una falta de relación sintáctica y léxica que interrumpe la continuidad semántica y la identificación de los sujetos, lo que está muy ligado con la manera en que funciona la LS mexicana.

\section{Lingüística sistémico-funcional}

La lingüística sistémico-funcional (LSF) de Halliday (2014) es el modelo escogido en este artículo para analizar las producciones escritas de las personas Sordas, porque se enfoca en "la descripción de la lengua como un recurso para significar más que como un conjunto de reglas" (Halliday y Martin, 1993, p. 22).

La LSF trabaja con textos completos y usa las cláusulas como unidad básica del estrato léxicogramatical (Halliday, 2014), porque es la construcción de un complejo de ideas que se relacionan entre sí para significar las cosas, los acontecimientos y los sentimientos. Las cláusulas se relacionan entre sí formando complejos de cláusulas de manera paratáctica e hipotáctica, lo que en otras teorías es equivalente a la coordinación y a la subordinación. 
A partir de la cláusula se manifiestan tres metafunciones simultáneamente: la lógica, la interpersonal y la textual. En esta investigación se trabajó con la metafunción lógica, para dar cuenta de los grupos, las frases y las clases de palabras que constituyeron las muestras. Para el análisis, también se utilizó la propuesta adaptada de la LSF para la lengua española, desarrollada por Ghio y Fernández (2005).

Halliday (2014) propone las siguientes unidades sintagmáticas:

- El grupo nominal (GN): conformado por la "cosa” de la que se habla, es decir, un nombre propio, común o pronombre, que puede ir acompañado de pre o posmodificadores (deícticos, posdeícticos, epítetos, clasificadores, numerativos y frases preposicionales).

- El grupo verbal (GV): el proceso dentro de la cláusula. Puede ir compuesto por un solo verbo, que expresa el evento, o expandirse a un operador finito y un predicador.

- El grupo adjetival (G. Adj): un adjetivo que funciona como núcleo. Para la investigación, solo se tomaron en cuenta los adjetivos que funcionaron como atributo en un proceso o con otros premodificadores y posmodificadores.

- El grupo adverbial (G. Adv): un adverbio que funciona como núcleo y puede estar acompañado de premodificadores y posmodificadores.

- El grupo conjuntivo (GC):una conjunción que une dos o más cláusulas o complejos de cláusula, y puede ser de tres clases: enlace, unión o continuativo.

- El grupo preposicional (GP): un grupo de preposiciones con más de una palabra.

- La frase preposicional (FP): un conjunto conformado por una preposición con función de núcleo, seguida de un GN. En la investigación solo se contabilizaron las FP que no estuvieran dentro del GN, con el fin de evitar doble registro.

\section{Aportes de otras investigaciones}

Se han realizado investigaciones sobre la lengua escrita de personas Sordas que sirven de base para el análisis. A continuación se mencionan algunas de ellas.

K. Tovar (2016) usa la LSF y manifiesta que los Sordos caleños tienen un grado bajo de comprensión de conjunciones, vocabulario, tiempos verbales, pronombres y algunos adjetivos. También Scott y Hoffmeister (2018) exponen que más de la mitad de los estudiantes estadounidenses hacen intentos por definir conceptos, pero no logran ser claros y precisos, como correspondería al inglés académico.

Por su parte, Herrera (2014) y Herrera et al. (2007) muestran que los chilenos manejan la estructura discursiva narrativa, pero usan oraciones simples y con errores. Igualmente, Morales (2009) expone que los Sordos identifican la macroestructura de los tipos textuales, pero desconocen aspectos como género, número, conjugación verbal, artículos, sustantivos, adjetivos, complementos, vocabulario, sinónimos, antónimos y diminutivos.

Por último, Gutiérrez (2012) analizó la escritura de alumnos Sordos españoles de primaria y secundaria, observando que a pesar de que hay organización discursiva, les falta dominio de recursos lingüísticos formales, lo que afecta la calidad de la organización textual.

\section{Método}

Se hizo un análisis descriptivo de las unidades sintagmáticas presentes en textos escritos por estudiantes Sordos, utilizando la LSF. También se llevó a cabo una entrevista para obtener información sociolingüística y percepciones de los participantes respecto a su aprendizaje del español como L2.

A través de estos procesos, se respetaron las normas éticas de recolección y tratamiento de los datos, y se obtuvo el consentimiento informado de los participantes y sus acudientes.

Los siguientes párrafos describen con mayor detalle los participantes y sus características, los instrumentos utilizados para recolectar los datos y el método para llevar a cabo su análisis. 


\section{Los participantes}

En el estudio participaron cuatro estudiantes Sordos, correspondientes al total de matriculados en el último año escolar (grado 11. ${ }^{\circ}$ ) de una institución educativa pública de Cali, Colombia, que trabaja bajo el modelo de educación bilingüe bicultural.

Los estudiantes tenían entre 19 y 20 años, hipoacusia neurosensorial bilateral profunda, a excepción de uno, de quien no se pudo conocer el grado de pérdida. Recibieron terapia oralista durante la infancia. Al momento de la investigación usaban ayudas auditivas, aunque no con la misma frecuencia e intensidad. La LSC era su L1 y estaban en proceso de adquisición del español escrito como L2 dentro de su contexto escolar.

\section{Instrumentos y recolección de datos}

Los datos se recolectaron dentro de la institución educativa mediante textos escritos por los estudiantes y entrevistas, con el acompañamiento de la intérprete de LSC encargada del grado 11 . $^{\circ}$.

Se recogieron 16 textos. Para ello, la investigadora diseñó cuatro talleres, cada uno de 80 minutos de duración, aplicados a lo largo de un mes, uno por semana. Los temas se relacionaron con cultura Sorda y fútbol, por lo que eran reconocidos por los participantes.

Se usaron videos para presentar los temas y se dieron consignas en LSC para que luego escribieran manualmente y de manera independiente, en hojas en blanco.
No se brindó modelamiento, es decir, ejemplos de cómo escribir, pero se permitió a los participantes hacer preguntas.

Finalmente, cada estudiante escribió dos textos narrativos y dos explicativos. Con esto se buscó obtener producciones en las que se viera el uso léxico-gramatical del español que hacen cuando narran y explican mediante sus propios recursos lingüísticos, ya que son acciones propias del contexto académico.

También se realizó una entrevista semiestructurada individual sobre la historia sociolingüística, basándose en Finau (2014) y Santos (2009), y sobre aspectos afectivos relacionados con la L2 (Brown, 2007). La entrevista se efectuó en LSC, duró 20 minutos y se grabó en video.

\section{Análisis de los datos}

Los 16 textos fueron tratados como productos, partiendo del hecho de que la escritura debe entenderse en sí misma. Por eso, no se pidió explicación sobre lo que escribieron; además, no había manera de asegurar que recordaran por qué habían tomado determinadas elecciones.

Cada texto se transcribió conservando la forma original en que fueron escritos por los participantes y se dividió manualmente en cláusulas, usando las convenciones de división propuestas por Halliday (2014, p. 10), que pueden observarse en la Tabla 1. Para determinar el límite de cada cláusula, se identificó el GV, que es el elemento

Tabla 1 Convenciones de notación para representar la constitución léxico-gramatical de las cláusulas

\begin{tabular}{llllll}
\hline Convención & \multicolumn{1}{c}{ Rango } & Convención & \multicolumn{1}{c}{ Rango } & Convención & \multicolumn{1}{c}{ Rango } \\
\hline|| $\mid$ & Complejo de cláusula & {$[[[]]$} & $\begin{array}{l}\text { Complejo de cláusula bajada } \\
\text { de rango }\end{array}$ & $<<<>>>>$ & $\begin{array}{l}\text { Complejo de cláusula } \\
\text { incrustado }\end{array}$ \\
|| & Cláusula & {$[[]]$} & Cláusula bajada de rango & $<<>>$ & Cláusula incrustada \\
$\mid$ & Grupo o frase & {[]} & Grupo o frase bajada de rango & $<>$ & Grupo o frase \\
\end{tabular}


mínimo que la conforma, y en los casos en que no estuvo presente, se tomó como base el GN. Se aclara que cuando uno de los dos elementos fue omitido en las cláusulas, lo que dificulta su comprensión, la división fue ejecutada según el criterio de la investigadora.

A continuación se observa un ejemplo de cómo se hizo este procedimiento con un texto de un participante:

|||[[Porque el problema de comunicar entre oyentes y Sordos es dificulta de comunicarse]] y a oyente habla mas que otró oyente [[para comunicarse bien,]] y el oyente te ignora a Sordo, [[porque lo Sordo no puede hablar la voz]] es que le gusta mas las señas y [[porque falta la palabra correcta.]] |||

|||De sluccionar de oyente a Sordo [[para que aprendé la señas|| para enseñarla]] ||y luego el oyente le gusta el abecedario de la letra \|y el diferente los mas dificil de la palabra señas|| y el oyente queda paciencia [[para cambiar las señas [[para comunicarse bien entre oyente y Sordo]]]], y el oyenté va hacer gesticular [[para que los Sordos entienda bien]] y los Sordo tambien va empatia de la palabra correcta |ly alguno la palabra la incorrecta ||y el oyente va ayudar a corregir la palabra [[para que se queda bien perfecto:)]] |||

A su vez, cada cláusula se dividió en GP y FP, mediante la escala de rangos de Halliday (2014). De esta manera, se obtuvo información que se procesó en Excel $^{\circledR}$, con el fin de obtener datos cuantitativos, como se observa en las primeras dos tablas del siguiente apartado.

También se usó el programa AntConc (Laurence, 2014) para obtener listas y frecuencias de palabras y colocaciones, con el fin de observar tendencias; sin embargo, de estas últimas no se encontraron datos significativos, porque los participantes no necesariamente usaban las palabras con la misma función que tienen en español. Por eso, no se estimó totalmente eficaz el uso de programas digitales para procesamiento de corpus, ya que las etiquetas están diseñadas para la lengua estándar.

Adicionalmente, se contabilizaron los siguientes tipos de divergencias: del GV, cuando había omisión de un verbo del grupo, conjugación incoherente con el tiempo o el número de la "cosa"; del GN, cuando la "cosa" o todo el grupo era elidido o si no había correspondencia entre los deícticos y la "cosa"; de los deícticos determinantes, cuando eran omitidos o usados innecesariamente en una cláusula; de FP, cuando se omitía una preposición que precedía a una frase que funcionaría como posmodificador de una "cosa" o de un adverbio. Por último, bajo la etiqueta "Otras observaciones" se clasificaron hallazgos donde hubo omisión de un grupo o parte del mismo, uso excesivo de conjunciones, ordenación alterada de grupos en una cláusula, presencia innecesaria o ausencia equívoca de palabras o grupos en una cláusula, y cláusulas que generaban mucha dificultad para comprender el sentido de un complejo de cláusulas.

Luego de este proceso, como la investigadora tiene un nivel básico de LSC, solicitó el apoyo de un intérprete de LSC y licenciado en Español, para resolver inquietudes que surgieron en apartados difíciles de entender y aclarar dudas de posibles transferencias entre la LSC y el español.

Finalmente, se transcribieron los datos de la entrevista con ayuda del intérprete y se hizo una caracterización sociolingüística de los estudiantes y de aspectos afectivos que pueden influir en la producción del español.

\section{Resultados}

Las entrevistas mostraron que los participantes tenían diferentes niveles de LSC, con la que se contactaron por primera vez cuando uno de ellos tenía 6 años de edad, mientras que los demás lo hicieron después de los 10 años. Todos recibieron terapia oralista, pero al momento de la investigación habían elegido la LSC como L1. Todos tuvieron contacto con el español escrito después de los 8 años de edad.

Se indagó también sobre las formas de comunicarse en los contextos inmediatos. En la escuela tenían una persona Sorda como modelo lingüístico, que dictaba la clase de cultura Sorda, y una 
intérprete de LSC, para todas sus clases con docentes y compañeros oyentes. La docente que les dictaba la clase de español era bilingüe en español e inglés, pero no sabía LSC, ni tenía formación en educación de personas Sordas.

En cuanto a su hogar, dos estudiantes se comunicaban oralmente con sus familias, ya que habían logrado algún nivel de oralización en la infancia; otro tenía una familiar que conocía algunas señas y el último contaba con un hermano menor que sabía LSC.

Adicionalmente, tres de los participantes manifestaron que aparte de la intérprete, el modelo lingüístico y la clase de español, no recibían más apoyo para el aprendizaje de ninguna de las lenguas, y uno refirió recibir algún apoyo de su familia.

Por otro lado, tres de los cuatro participantes dijeron tener motivación por aprender español hasta alcanzar un nivel que les facilitara comunicarse inde-

22 pendientemente y estudiar una profesión, aunque consideraban que, de cualquier forma, los intérpretes siempre serán necesarios para la comunidad Sorda. Uno de ellos no mostró interés y no se pudo conocer la razón al preguntarle, pero la intérprete explicó que tal vez el estudiante no tenía suficiente desarrollo en ninguna lengua para explicarlo.

Al indagar por sus hábitos de lectura, solo uno de los estudiantes manifestó tenerlo.

Sobre su experiencia con el español, dijeron que era difícil de aprender, especialmente la conjugación verbal, la estructuración y la cohesión entre párrafos, mientras que les parecía más sencillo el vocabulario relacionando con nombres y adjetivos.

Por otra parte, de los textos se puede afirmar que el análisis lingüístico fue dispendioso, en cuanto la léxico-gramática hallada fue distante de la lengua estándar, por lo que la interpretación de los datos dependió, en cierta medida, de las hipótesis sobre los usos que los participantes hicieron de la L2 y del análisis de las divergencias realizado entre la investigadora y el intérprete de LSC.
En la Tabla 2 se puede observar el total de cláusulas de la muestra y se detalla cuántas produjo cada participante por texto.

En relación con la estructuración general de las cláusulas, algunas conservaban la léxico-gramática del español, especialmente en el caso de dos de los participantes; sin embargo, en todos los textos hubo mayor producción de cláusulas que no son fáciles de comprender, debido a divergencias como falta de conjunciones entre ellas, ausencia de una cláusula que se requeriría para organizar mejor un complejo de cláusulas, ubicación de los grupos en orden diferente al de la lengua estándar, entre otros.

En cuanto a las unidades sintagmáticas (véase Tabla 3), se identificaron mayormente GN, seguidos de GV y de FP, mientras que los GP, G. Adj y G. Adv no estuvieron presentes en todos los textos. Los GP fueron muy escasos.

Específicamente, en los 332 GN, se identificaron los que estaban compuestos solo por núcleo o que además tenían premodificadores o posmodificadores, como se muestra en algunos ejemplos en la Tabla 4. No se encontraron GN con deícticos de tipo determinante inclusivo, numerativos ordenativos exactos ni inexactos, y tampoco fueron de uso común el marcador no genitivo ni el marcador genitivo, que solo fue detectado una vez. Como se dijo anteriormente, los ejemplos ${ }^{1}$ conservan la forma original en que los produjeron los participantes y en las tablas han sido separados por comas.

El GN fue el segundo grupo con más divergencias, puesto que estas se hallaron desde el $20 \%$ en los textos del participante que menos las produjo, hasta el $48 \%$ en los del participante que más las escribió. Aquellas se caracterizaron por presentar

1 Todos los ejemplos del artículo están escritos en cursiva para evitar confusiones, porque al ser en español, es necesario diferenciarlos visualmente de la escritura de la autora. 
omisión del núcleo o de todo el grupo en la cláusula; falta de correspondencia entre la "cosa" y el género o el número de un premodificador o posmodificador; reemplazo de la "cosa" por otra palabra con la misma función, pero que para el contexto de la cláusula no se consideró pertinente, y alteración del orden del GN, como se puede ver en algunos ejemplos de la Tabla 5.

Tabla 2 Contabilización de las cláusulas

\begin{tabular}{lccccc}
\hline \multicolumn{1}{c}{ Participante } & $\begin{array}{c}\text { Cláusulas } \\
\text { del texto } \\
\text { expositivo 1 }\end{array}$ & $\begin{array}{c}\text { Cláusulas } \\
\text { del texto } \\
\text { expositivo 2 }\end{array}$ & $\begin{array}{c}\text { Cláusulas } \\
\text { del texto } \\
\text { narrativo 1 }\end{array}$ & $\begin{array}{c}\text { Cláusulas } \\
\text { del texto } \\
\text { narrativo 2 }\end{array}$ & $\begin{array}{c}\text { Total de } \\
\text { cláusulas por } \\
\text { participante }\end{array}$ \\
\hline Estudiante 1 & 22 & 14 & 37 & 22 & 95 \\
Estudiante 2 & 22 & 26 & 22 & 28 & 98 \\
Estudiante 3 & 23 & 43 & 24 & 35 & 125 \\
Estudiante 4 & 18 & 32 & 30 & 19 & 99 \\
Total de cláusulas por texto & 85 & 115 & 113 & 104 & 417 \\
\hline
\end{tabular}

Tabla 3 Unidades sintagmáticas

\begin{tabular}{lccccccc}
\hline $\begin{array}{c}\text { Participante/ } \\
\text { Unidad } \\
\text { sintagmática }\end{array}$ & $\begin{array}{c}\text { Grupo } \\
\text { nominal } \\
\text { (GN) }\end{array}$ & $\begin{array}{c}\text { Grupo } \\
\text { verbal } \\
\text { (GV) }\end{array}$ & $\begin{array}{c}\text { Grupo } \\
\text { adjetival } \\
\text { (G. Adj) }\end{array}$ & $\begin{array}{c}\text { Grupo } \\
\text { adverbial } \\
\text { (G. Adv) }\end{array}$ & $\begin{array}{c}\text { Grupo } \\
\text { conjuntivo } \\
\text { (GC) }\end{array}$ & $\begin{array}{c}\text { Grupo } \\
\text { preposicional } \\
\text { (GP) }\end{array}$ & $\begin{array}{c}\text { Frase } \\
\text { preposicional } \\
\text { (FP) }\end{array}$ \\
\hline Estudiante 1 & 94 & 71 & 2 & 12 & 15 & 0 & 19 \\
Estudiante 2 & 77 & 83 & 7 & 13 & 19 & 2 & 25 \\
Estudiante 3 & 87 & 87 & 2 & 5 & 18 & 0 & 31 \\
Estudiante 4 & 74 & 73 & 7 & 6 & 20 & 0 & 32 \\
Total & 332 & 314 & 18 & 36 & 72 & 2 & 107 \\
\hline
\end{tabular}

Tabla 4 Ejemplos de grupos nominales

\begin{tabular}{|c|c|c|}
\hline $\begin{array}{l}\text { Grupo nominal con } \\
\text { premodificadores }\end{array}$ & $\begin{array}{l}\text { Grupo nominal con } \\
\text { posmodificadores }\end{array}$ & $\begin{array}{l}\text { Grupo nominal con pre y } \\
\text { posmodificadores }\end{array}$ \\
\hline $\begin{array}{l}\text { Los soluciones, la universidad, la casa, la } \\
\text { señas, los arbitro, los sordos, el oyente, } \\
\text { la voz, mi mamá, } 7 \text { años, mi familia, buen } \\
\text { disdipina }\end{array}$ & $\begin{array}{l}\text { medias blanco, azul, amarilla; normas de } \\
\text { futbol, ley de normas, camisa de futbol, } \\
\text { entrada fuerte, comunicacion difícil, red } \\
\text { social, comunidad sordos }\end{array}$ & $\begin{array}{l}\text { Muchos problemas de fiebre, la persona } \\
\text { sorda, la lengua de señas, una pasta de } \\
\text { dolor "Genizol" acetaminofen, la tarjeta } \\
\text { amarilla, el abecedario de la letra, un } \\
\text { maestro de Sordo }\end{array}$ \\
\hline
\end{tabular}

Tabla 5 Ejemplos de divergencias del grupo nominal

\begin{tabular}{lccc}
\hline $\begin{array}{c}\text { Omisión del grupo } \\
\text { nominal o su núcleo }\end{array}$ & $\begin{array}{c}\text { Cláusulas en que } \\
\text { sobra elemento del } \\
\text { grupo nominal }\end{array}$ & $\begin{array}{c}\text { Cláusulas en las que el } \\
\text { orden del grupo nominal } \\
\text { está alterado }\end{array}$ & $\begin{array}{c}\text { Cláusula con } \\
\text { divergencia de número } \\
\text { o género }\end{array}$ \\
\hline $\begin{array}{l}\text { Mejorar es enseñará penal, ya maestro yo enseñas en } \\
\text { Que hace muy dificl, Deber } \\
\text { de hacer una regla }\end{array}$ & $\begin{array}{c}\text { Es la cabeza de dolor, lo que es sorda profundo, fui al } \\
\text { necesitaba una pasta de dolor }\end{array}$ & \begin{tabular}{l} 
farmaceuitca, las seña \\
\hline
\end{tabular} \\
\hline
\end{tabular}


Tabla 6 Ejemplos de grupos verbales

\begin{tabular}{lcc}
\hline $\begin{array}{c}\text { Grupo verbal constituidos } \\
\text { únicamente por núcleo }\end{array}$ & $\begin{array}{c}\text { Grupo verbal compuesto por Operador } \\
\text { Finito y Predicador }\end{array}$ & $\begin{array}{c}\text { Grupo verbal con "PolaRIDAD" } \\
\text { negativa }\end{array}$ \\
\hline $\begin{array}{l}\text { prenguto, nacio, fue, demora, } \\
\text { escribe, enseña, es. }\end{array}$ & $\begin{array}{l}\text { empujar debe, puede jugar, no puedo estudia, no sabe, no puedo, no creo, no puedo } \\
\text { fui va, sabe bien escribir, puede claro entiende, pedir, no usa, no puedo hacer, no } \\
\text { va hacer gesticular, va ayudar a corregir, }\end{array}$ & puede quitar \\
\hline
\end{tabular}

Tabla 7 Ejemplos de divergencias de los grupos verbales

\begin{tabular}{llll}
\hline \multicolumn{1}{c}{ Omisión de verbo } & \multicolumn{1}{c}{ Conjugación verbal } & Omisión de un componente & $\begin{array}{c}\text { Adición innecesaria de un } \\
\text { verbo }\end{array}$ \\
\hline $\begin{array}{lll}\text { Sordo difícil comunicación, } \\
\text { Porque no faccil, Como la habla con oyente, } \\
\text { tarjeta amarilla. }\end{array}$ & $\begin{array}{l}\text { Ne slucionar de oyente a Sordo, como llamar es mi Hermana, } \\
\text { primera vez no sabe }\end{array}$ & $\begin{array}{l}\text { la palabra correcta y alguno la señas } \\
\text { palabra la incorrecta }\end{array}$ \\
\hline
\end{tabular}

La segunda unidad sintagmática con más número de apariciones fue el GV, con 314 hallazgos. Se identificaron GV constituidos por núcleo, por un Operador Finito y un Predicador o denotando "POLARIDAD" negativa o positiva, como se muestra en la Tabla 6.

De todos los grupos, en el GV fue en el que se halló el mayor número de divergencias, desde el $38 \%$ en los GV del participante que menos las produjo, hasta el $75 \%$ en el participante que más las hizo. Las divergencias estuvieron relacionadas con la conjugación verbal, la omisión de todo el GV o de uno de sus componentes, el uso de un verbo que no era el más adecuado para el contexto o el uso de verbos que sobraban en la cláusula, como puede verse en los ejemplos de la Tabla 7.

Por otra parte, se encontraron $18 \mathrm{G}$. Adj en once textos. La mayoría estuvieron conformados solo por núcleo y unos cuantos presentaban el adverbio muy como premodificador; por ejemplo: muy peor, muy famoso, muy dificil, muy fácil.

En lo referente al G. Adv, se detectaron 36 veces en 13 textos, siendo producidos en mayor medida por dos de los participantes. Estos se constituyeron únicamente por núcleo, como se aprecia en la parte subrayada en los siguientes ejemplos: demora mucho, sabe bien escribir, puede claro, haciendo trampa mucho tiempo, se queda bien perfecto, habla más, comunicarse bien, muy rápido, hablas como iempre, ya nacio. Y solo dos de estos grupos estaban constituidos por más de un adverbio, como se ve los ejemplos: aprendo poco a poco y poco a poco ya hay señas.

En cuanto al GC, se encontró 72 veces, en su mayoría constituido solo de núcleo. Los cuatro participantes usaron: $y$, pero, porque, para, siendo $y$ el más frecuente. En cuanto a grupos compuestos por más de una conjunción, se hallaron: $y$ luego, $y$ entonces. Otros GC mucho menos frecuentes usados por uno o dos de los participantes fueron: $n i$, ejemplo, así, si, entonces y mas.

Por último, el GP fue el de menor ocurrencia, producido dos veces por un solo participante, mediante el grupo dentro de, mientras que las FP fueron escritas 107 veces en textos de todos los participantes. Sobre las FP, hubo divergencias por omisión de la preposición, que daría paso a un posmodificador de la "cosa" en un GN, como, por ejemplo: Como empatía comunicacion comunidad Sordos, Kruse van la ciudad de Sch leswig; o, por el contrario, por el uso de una preposición que no se consideró pertinente en la cláusula o que reemplazaba a otra que sería más adecuada, como en el 
caso del ejemplo: Fue en la escuela de oyentes, nuevos jugadores de la primera vez.

\section{Discusión}

Los datos de las entrevistas permiten reflexionar sobre la necesidad de seguir promoviendo el contacto temprano con la LSC para las familias de personas Sordas, pues se sigue invirtiendo parte importante de la primera infancia en procesos de oralización, sin propiciar de la misma forma el contacto con la lengua de la comunidad Sorda y, como se puede observar, todos los participantes optaron con el tiempo por hacer de la LSC su L1.

Además, como lo explica Herrera (2014), la mayoría de las personas Sordas ingresan al sistema escolar sin una L1 consolidada y deben aprender a leer y escribir en una segunda lengua que no conocen y a la que no se pueden exponer de manera natural en sus primeros años de vida, a diferencia de los estudiantes oyentes bilingües. Igualmente, Scott y Hoffmeister (2018) plantean que dominar la LS es un fuerte predictor para alcanzar precisión en la escritura académica.

También es necesario revisar cómo los oyentes están promoviendo la adquisición de la L2 de los estudiantes Sordos, pues la mayoría de personas que los rodean no tienen una comunicación fluida cara a cara en LSC, lo que disminuye las oportunidades para interactuar en tiempo real y orientar la comprensión de actividades personales o propias de esferas donde el español es dominante, como lo son las tareas escolares. Por tanto, teniendo en cuenta los aportes de Brown (2007), puede que esto genere problemas sobre aspectos afectivos influyentes en la adquisición de la L2, como la empatía y el deseo de comunicarse entre oyentes y Sordos, y la autoestima de los aprendices Sordos como minoría lingüística. En este orden de ideas, Noel (2019) afirma que la educación de los Sordos requiere que en todo momento se viva en un ambiente de bilingüismo.

Sumado a lo anterior, debe aprovecharse la motivación de los participantes para comunicarse en español, pues como lo explica Galvis (2005), influye en el desarrollo de la interlengua y la conciencia metalingüística y metacognitiva. Además, ello impacta el valor que los aprendices dan a la L2, los hace recursivos y determina el grado de cumplimiento de sus metas (Brown, 2007).

Por eso es muy importante que los educadores oyentes interactúen en LSC y conozcan sobre bilingüismo, interculturalidad y metodologías para enseñar L2 de diferente modalidad (Herrera y Paz, 2019; Muñoz, 2020; Noel, 2019). Al respecto, Mayer y Trezek (2019) afirman que, en la actualidad, hay docentes que manifiestan no sentirse preparados para enseñar la escritura a los estudiantes Sordos. Y Williams y Mayer (2015) explican que la LS es necesaria, pero no suficiente, porque también se precisa la adquisición del principio alfabético, el desarrollo de la sintaxis y la gramática de la L2.

Por otra parte, en lo concerniente al análisis de los textos escritos, la alta frecuencia de cláusulas difíciles de comprender es un elemento común en los textos de la comunidad Sorda, ya que, como lo manifiestan Herrera (2014), Mayer y Trezek (2019), Rusell (2016) y Scott y Hoffmeister (2018), la sintaxis de la L2 es uno de los aspectos más difíciles. Lo mismo manifestó Ruiz (2011), al afirmar tener problemas para comprender los límites entre cláusulas escritas por esta población, porque en su escritura no había correspondencia término a término con las estructuras del español o había problemas con la puntuación, lo cual resultaba incoherente para quien no supiera lengua de señas mexicana.

Adicionalmente, Galvis (2005) explica que cuando el grado de competencia en la L2 no es suficiente, se observa transferencia de la LSC en los escritos, por lo que es de esperarse que no haya correspondencia palabra-seña y en ocasiones recurran a la perífrasis y la paráfrasis, o que una idea completa en español pueda expresarse con pocas señas y, al escribirla, sus estructuras sean simplificadas. 
Sin embargo, no se puede negar que, a pesar de la influencia de la LSC en el español, los estudiantes se esfuerzan por utilizar elementos del español, en algunos casos con más acierto que otros. Como lo expresan Massone et al. (2005), no es cierto que las personas Sordas utilicen una estructura pura de la LS al escribir en español, pues agregan a sus textos elementos morfológicos y léxicos que no están presentes en la Ls y utilizan signos ortográficos, lo cual concuerda con las características de la interlengua expuestas en la literatura (Larsen-Freeman y Long, 1991). En el caso del inglés, el $60 \%$ de los estudiantes intentan definir términos, pero sin la precisión propia del registro académico (Scott y Hoffmeister, 2018).

En cuanto a las unidades sintagmáticas, la composición de los GN coincide con lo observado por K. Tovar (2016), Massone et al. (2005) y Zambrano (2008), quienes también reportaron falta de correspondencia entre género y número, y 26 omisión de artículos, que en la LSF han sido denominados “deícticos". Además, las divergencias del GN pueden estar relacionadas con que las personas Sordas muchas veces desconocen que una misma seña tiene diferentes nombres en español y utilizan los que conocen, pudiendo generar un significado diferente al que esperan, sin ser conscientes, aspecto en el que no difieren de los aprendices oyentes de lenguas orales. Lissi et al. (2012) tratan estos casos como dificultades semánticas, al igual que Galvis (2005), quien desde la visión de la interlengua las denomina "transferencias semánticas".

Sumado a lo anterior, se debe tener en cuenta que en LSC, los nombres propios suelen representarse por medio de una seña icónica, lo que hace que no se practique su deletreo constantemente; por lo tanto, es natural que se presenten inversiones, migraciones o reemplazos de grafemas, como, por ejemplo, nalpon (Napoleón), matemacias (matemáticas) y geofitica (geografía).

Pasando a otro punto, los GV guardan relación con lo manifestado en las entrevistas acerca de lo difícil que es para los participantes la conjugación verbal y puede tener una explicación si se considera que el español ha sido influido por la LSC, debido a que esta no posee señas para conjugar cada verbo, sino que los produce en infinitivo, y mediante rasgos no manuales y marcaciones espaciales se indica su tiempo, persona, "POLARIDAD" y otras características.

Esto concuerda con la explicación de Menéndez (2012) sobre las divergencias que observó en la conjugación verbal de la L2 de personas Sordas, manifestando que había influencia importante de la LS, en la que la concordancia verbal depende de relaciones espaciales y visuales, y no de relaciones gramaticales, como en el español.

Las divergencias en la conjugación verbal de los GV se asemejan a las encontradas por Galvis (2005), Morales (2009), Ruiz (2011) y Zambrano (2008). En especial, Ruiz (2011) afirma que la falta de marcación de la persona y el número en los verbos genera problemas en lo más básico de la cohesión, puesto que la concordancia entre sujeto y verbo ancla el tópico primario en la cláusula y expone que, al igual que en la presente investigación, tuvo que recurrir a la deducción para reconocer los sujetos por medio del contexto de las cláusulas y las narraciones. Adicionalmente, deja claro que el sistema de conjugación del español es difícil para todos los aprendices de L2, ya sean oyentes o Sordos, lo que genera muchos problemas en la interlengua.

En cuanto al G. Adj, al hacer el análisis en compañía de intérprete de LSC, se plantea que su baja producción se puede relacionar con las diferencias que hay entre la LSC y el español, ya que este corresponde a una lengua lineal, en la que se observa cada componente de la cláusula en un espacio y en la que se utiliza el verbo es para establecer relación de atributo entre la "cosa" y el adjetivo; sin embargo, no pasa lo mismo en LSC, que es una lengua tridimensional, sin seña específica para el verbo es para relaciones de atributo y solo se habla de G. Adj en LSC cuando se marca posesión. 
Asimismo, otros estudios reportaron omisión especial del verbo copulativo (proceso relacional, en LSF) "ser/estar". Específicamente, Menéndez (2012) explica que en Ls, la cópula ser/estar no aparece de manera explícita. Por su parte, Ruiz (2011) manifiesta que esto puede ser un marcador importante de la interlengua, por transferencia de la LS a la L2. En la misma línea de ideas, Galvis (2005) y $\mathrm{K}$. Tovar (2016) reportan que dicha omisión en la L2 es parte de un fenómeno de simplificación propia del proceso de interlengua. Adicionalmente, Massone et al. (2005) exponen que la lengua de señas argentina, así como en muchas otras LS, las cualidades de las personas y de los objetos son verbos de estado y no hay adjetivos, por lo que al ser un sistema diferente al español, genera inseguridades a la hora de ser usado en la escritura. L. Tovar (2008) también especula sobre esta posibilidad.

Teniendo en cuenta lo anterior, se considera importante que las personas Sordas puedan comprender el significado de los G. Adj en diferentes contextos, puesto que en LSC son incluidos mediante rasgos no manuales y muchos no son usados, por lo que existe la posibilidad de que no sean conscientes de ellos al momento de escribir en español.

En cuanto a los GC, en su mayoría los participantes optaron por un repertorio limitado, con alta frecuencia de la conjunción $y$. Al respecto, L. Tovar (2008) señala que las personas Sordas no suelen usar este tipo de grupos, ya que en su repertorio no existen muchas de las conjunciones del español o las que hay son préstamos de este, puesto que la LSC, por ser una lengua de comunicación cara a cara, naturalmente hace más uso de continuativos gestuales (en vez de orales) que de conjunciones.

Adicionalmente, el intérprete de LSC que colaboró en el análisis anotó que muchas personas Sordas escriben cláusulas separadas o enumeradas, evitando las conjunciones que relacionan párrafos; por lo tanto, propuso la hipótesis de que puede ser más más fácil escribir textos expositivos que narrativos, aun cuando este último sea más sencillo de producir en LSC que el primero.
En lo relacionado con los GP y FP, los hallazgos coinciden con los de Massone et al. (2005) y se consideran naturales si se tiene en cuenta la interlengua de los estudiantes, puesto que, como explica Menéndez (2012), en las LS se observa frecuente omisión de preposiciones, porque no siempre son necesarias en el discurso, debido a que por su carácter icónico muchas veces las estructuras de los clasificadores permiten mostrar la posición, la forma y el movimiento en el espacio. Además, L. Tovar (2008) explica que en la LSC no hay FP y Galvis (2005) añade que, en la escritura, las preposiciones y otras palabras funcionales son omitidas, por cuanto en el proceso de interlengua se simplifican estructuras por desconocimiento de la L2.

\section{Conclusiones}

Gracias al análisis hecho con la LSF se pudo determinar que los estudiantes Sordos se encuentran en un proceso de interlengua, en el que se observan elementos de la LSC y otros propios del español, como GN con premodificadores tales como deícticos, y con posmodificadores de tipo FP; GV con algunas conjugaciones adecuadas, varios GC, etc. Esto puede ser una base para continuar desarrollando estrategias pedagógicas que permitan establecer objetivos específicos relacionados con el desempeño en la léxico-gramática de la L2.

También es necesario entender el papel que desempeñan los oyentes que rodean a los estudiantes Sordos en sus contextos escolar y familiar, pues aunque cada uno tiene características sociolingüísticas diversas, comparten situaciones sobre las que se pueden tomar decisiones para mejorar su proceso bilingüe. La primera es que pueden recibir la clase de español con un docente usuario de LSC, conocedor de la cultura Sorda y experto en educación bilingüe. La segunda es que los familiares oyentes pueden aprender LSC para propiciar una comunicación más fluida en casa. Y la tercera es que, desde casa, se puede propiciar un hábito de la lectura que contribuya a brindar mayor estímulo de la L2. 
En relación con lo anterior, se resalta la importancia de comprender que los aspectos afectivos del bilingüismo cumplen un papel clave en la educación bilingüe; por eso, se reitera la necesidad de formar y disponer de profesores de español como L2 para Sordos con conocimiento de la LSC y cultura Sorda, ya que esto puede ser beneficioso para aprovechar la relación entre la LSC y el nivel de interlengua de cada estudiante, con lo que se evita calificar sus textos desde el "error", al reconocer que se trata de productos de personas bilingües que no necesariamente han terminado de consolidar ambas lenguas.

Por otra parte, entre las divergencias halladas en las unidades sintagmáticas hubo algunas que no obstaculizaron la comprensión del discurso, como es el caso de la falta de concordancia de algunos premodificadores con la "cosa" del GN. No obstante, las divergencias en los GV, GC y en la FP sí causaron dificultades mayores para entender el sentido de las cláusulas, lo que generaba ambigüedades. Aunque esto podría tratar de solucionarse preguntando a cada Sordo en LSC qué quiso decir, no es una salida viable como ejercicio permanente en la vida escolar cotidiana, ya que sería dispendioso y no necesariamente preciso. Por tanto, se sugiere trabajar sobre este último grupo de divergencias con especial atención.

En la misma línea de ideas, los estudiantes Sordos usaron poco el G. Adj, ya que, como se ha explicado, este no es igual en la LSC que en el español; por eso, se debe hacer un trabajo que les permita entender cómo y cuándo usarlo, lo que a su vez implica que aprendan a producir relaciones de atributo con los verbos ser-estar, que tampoco son comunes en su L1, mientras que sí abundan en español.

Finalmente, el bilingüismo de las personas Sordas no requiere que los textos estén totalmente escritos en español estándar para que sus producciones tengan sentido; es decir, no se trata de hacer esfuerzos en busca de un estudiante que escriba como un monolingüe ideal, sino de acercarlo cada vez más a producciones inteligibles, con menor ambigüedad y más competencia lingüística, recordando siempre que los contextos y los actores deben ser facilitadores del bilingüismo en situaciones de contacto entre dos culturas: la oyente y la sorda.

\section{Referencias}

Appel, R. y Muysken, P. (2005). Language contact and bilingualism. Amsterdam University Press - Amsterdam Academic Archive.

Brown, D. (2007). Principles of language learning and teaching. (5. ${ }^{\mathrm{a}}$ ed.). Pearson Education.

Carvajal, M. (2009). Dos comunidades, dos lenguas: planificación lingüística de los usos de la lengua de señas colombiana en un escenario educativo. Lenguaje, 37(1), 113-133. https://doi.org/10.25100/lenguaje.v37i1.4886

Celemín, J. C. (2014). Calidad educativa y Pruebas SABER 11: el caso de los estudiantes Sordos en tres colegios de Bogotá [Tesis de maestría]. Universidad Nacional de Colombia. https://repositorio.unal.edu.co/handle/ unal/54672

Colombia, Ministerio de Educación Nacional e Instituto Nacional para Sordos. (2019). Plan Estratégico Institucional INSOR 2019-2022. https://www. insor.gov.co/home/planeacion-gestion-y-control/ planeacion-institucional/planeacion-2019/

Finau, R. (2014). Aquisição de escrita por alunos surdos: a categoria aspectual como um exemplo do processo. Revista Brasileira de Linguistica Aplicada, 14(4), 935-956. https://doi.org/10.1590/ S1984-63982014005000023

Flórez, R., Baquero, S. y Sánchez, L. (2010). Desarrollo de habilidades en español escrito en personas sordas universitarias: estudio de caso. Forma y Función, 23(2), 1-28. https://revistas.unal.edu.co/index. $\mathrm{php} /$ formayfuncion/article/view/23851

Galvis, R. (2005). La interlengua de los estudiantes sordos en el aprendizaje del castellano escrito como segunda lengua. Pedagogía y Saberes, (22), 77-82. https:// doi.org/10.17227/01212494.22pys77.82

Ghio, H. y Fernández, M. (2005). Manual de lingüistica sistémico funcional. El enfoque de M.A.K. Halliday y R. Hasan: aplicaciones a la lengua española. Ediciones Universidad Nacional del Litoral.

Gutiérrez, R. (2012). Cohesión textual en la expresión escrita de alumnos sordos de educación primaria y secundaria: estudio descriptivo. Revista de Logopedia, 
Foniatría y Audiología, 32(4), 171-178. https://doi. org/10.1016/j.rlfa.2012.05.005

Halliday, M. A. (2014). Halliday's introduction to functional grammar (4. ${ }^{a}$ ed.) (Revisado por Matthiessen). Routledge Taylor and Francis Group. https://doi. org/10.4324/9780203431269

Halliday, M. A. K. y Martin, J. R. (Eds.) (1993). Writing science: Literacy and discursive power (pp. 69-85). University of Pittsburgh Press.

Herrera, V. (2014). Alfabetización y bilingüismo en aprendices visuales. Aportes desde las epistemologías de Sordos. Educación y Educadores, 17(1), 135-148. https://doi.org/10.5294/edu.2014.17.1.7

Herrera,V.y Paz,V.dela(2019).Prácticas pedagógicasytransformaciones sociales. Interculturalidad y bilingüismo en la educación de sordos. Revista Latinoamericana de Educación Inclusiva, 13(1), 73-88. https://doi. org/10.4067/S0718-73782019000100073

Herrera, V., Puente A. y Ardila A. (2007). Códigos de lectura en sordos: la dactilología y otras estrategias visuales y kinestésicas. Revista Latinoamericana de Psicologia, 39(2), 269-286. http://www.scielo.org co/pdf/rlps/v39n2/v39n2a05.pdf

Larsen-Freeman, D. (2014). Another step to be taken Rethinking the end point of the interlanguage continuum. En Z. Han y Tarone, E. (Eds.), Interlanguage: Forty years later (pp. 203-220). John Benjamins. https://doi.org/10.1075/1llt.39.11ch9

Larsen-Freeman, D. y M. H. Long. (1991). An introduction to second language acquisition research. Routledge Taylor and Francis Group.

Laurence, A. (2014). AntConc (Versión 3.4.3) [Windows 3.5.0 Dev]. Universidad Waseda. https://www.laurenceanthony.net/software/antconc/

Lissi, M., Svartholm, K. y González, M. (2012). El enfoque bilingüe en la educación de sordos: sus implicancias para la enseñanza y aprendizaje de la lengua escrita. Estudios Pedagógicos, 38(2), 299-320. https://doi. org/10.4067/S0718-07052012000200019

Luna, A. (2014). ¿Educación inclusiva? Análisis del marco jurídico sobre el derecho de acceso y permanencia en la educación superior para las personas Sordas en la ciudad de Bogotá. Revista de Derecho Público, (33), 1-56. https://doi.org/10.15425/ redepub.33.2014.18

Massone, M., Buscaglia, V. y Bogado, A. (2005). Los sordos aprenden a escribir sobre la marcha. Lectura y Vida: Revista Latinoamericana de Lectura,
26(4), 1-17. https://dialnet.unirioja.es/servlet/ articulo?codigo $=1402953$

Massone, M., Simón, M. y Gutiérrez, C. (1999). Una aproximación a la lengua escrita en la minoría sorda. Lectura y vida: Revista Latinoamericana de lectura, 20(3), 24-33. http://www.lecturayvida.fahce.unlp. edu.ar/numeros/a20n3/20_03_Massone.pdf

Massone, M. y Machado E. M. (1994). Lengua de señas argentina. Análisis y vocabulario bilingüe. Edicial.

Mayer, C. y Trezek, B. (2019). Writing and deafness: State of the evidence and implications for research and practice. Education Sciences, 9(3), 185. https://doi. org/10.3390/educsci9030185

Menéndez, B. (2012). Caracterización psicolingüística de la interlengua de alumnos sordos multilingües/multimodales en su acceso al castellano (L2/3) como lengua escrita. Monografías marco ELE, 15, 93-123. https://slabank.talkbank.org/access/0docs/Menendez2012.pdf

Morales, A. M. (2009). Producción de textos escritos por escolares sordos. Letras, 51(79),159-190. http:// ve.scielo.org/scielo.php?script $=$ sci_arttext\&pid $=$ S0459-12832009000200006

Muñoz, K. (2020). Comunidad sorda. Desarrollo desde una nueva construcción social. Polyphōnia, 4(2), 218-234. https://revista.celei.cl/index.php/PREI/ article/view/208

Noel, D. (2019). Sordedad y sordera: su implicación en la cultura y en el desarrollo evolutivo psico y sociolingüístico en la educación bilingüe. Revista RETOS XXI, 3(1), 72-85. https://doi.org/10.33412/retosxxi.v3.1.2349

Pacheco, C. de y Anzola, M. (2011). Análisis de las pruebas de suficiencia en lengua escrita española de estudiantes sordos universitarios. Revista de Teoria y Didáctica de las Ciencias Sociales, (17), 115-126. https://www. redalyc.org/articulo.oa? $\mathrm{id}=65221619008$

Rodríguez, D. (2013). El silencio como metáfora. Una aproximación a la comunidad sorda y a su sentimiento identitario. Periféria: Revista de Recerca i Formació en Antropologia, 18(1), 1-27. https://doi.org/10.5565/ $\mathrm{rev} /$ periferia. 378

Romaine, S. (1996). El lenguaje en la sociedad. Ariel.

Ruiz, E. (2011). Cohesión en el discurso narrativo de escolares sordos [Tesis de maestría]. Universidad Autónoma de Querétaro. https://xdoc.mx/download/ cohesion-en-el-discurso-narrativo-escrito-de-escolares-sordos-5e372fe $8645 \mathrm{a} 3$ ?hash $=8930 \mathrm{fb} 11 \mathrm{fc} 31 \mathrm{f} 6$ $38870 \mathrm{e} 4 \mathrm{df5} \mathrm{db} 3 \mathrm{a} 2 \mathrm{fa} 4$ 
Rusell, G. (2016). La escritura en sordos. Una propuesta metodológica para trabajar la sintaxis y el léxico desde el enfoque de español como lengua segunda y extranjera [Tesis de doctorado]. Universidad Complutense de Madrid. https://eprints.ucm.es/id/ eprint/38881/1/T37682.pdf

Santos, F. M. (2009). Marcas da LIBRAS e indícios de uma interlíngua na escrita de surdos em língua portuguesa [Tesis de maestría]. Universidade Federal de Bahia. http://repositorio.ufba.br/ri/handle/ri/10970

Scott, J. y Hoffmeister, R. (2018). Superordinate precision: An examination of academic writing among bilingual deaf and hard of hearing students. The Journal of Deaf Studies and Deaf Education, 23(2), 173182. https://doi.org/10.1093/deafed/enx052

Selinker, L. (1972). Interlanguage. International Review of Applied Linguistics in Language Teaching, 10(1-4), 209-232. https://doi.org/10.1515/ iral.1972.10.1-4.209

Tovar, K. (2016). La comprensión de la conjunción en la lengua española escrita por parte de personas sordas usuarias de la lengua de señas colombiana [Trabajo de pregrado]. Universidad del Valle.

Tovar, L. (1995). Bilingüismo: una visión desde la perspectiva de los sordos. El Bilingüismo de los Sordos, 1(1), 20-24.

Tovar, L. (2002). Un enfoque interdisciplinario para la enseñanza de la lengua escrita a niños Sordos. En C.
Curcó, M. Colín, N. Groult, y L. Herrera (Eds.), Contribuciones a la lingüistica aplicada en América Latina (pp. 269-292). Centro de Enseñanza de Lenguas Extranjeras, Universidad Autónoma de México.

Tovar, L. (2004). La necesidad de planificar una norma lingüística en lengua de señas para usos académicos. Lengua y Habla, 8(1), 97-132. https://dialnet.unirioja.es/servlet/articulo?codigo $=4004006$

Tovar, L. (2005). La educación bilingüe para sordos en señas y castellano escrito: un caso colombiano. En I. M. Muñoz, G. Merma, R. Nogueira y A. Peidro (Eds.), Estudios sobre la lengua de signos española, Universidad de Alicante 2001 (pp. 249-259). Universidad de Alicante.

Tovar, L. (2008). Definición, denominación y procesos de formación de neologismos de la lengua de señas colombiana: contribución a su planificación lingüistica [Tesis de doctorado]. Universidad de Los Andes, Venezuela.

Williams, C. y Mayer, C. (2015). Writing in young Deaf children.Review of EducationalResearch, 85(4), 630-666. https://doi.org/10.3102/0034654314564882

Zambrano, L. (2008). Modelo integral de alfabetización para personas sordas (MIDAs). Investigación y Postgrado, 23(2), 205-240. http:// ve.scielo.org/scielo.php?script=sci_arttext\&pid $=$ S1316-00872008000200008

Cómo citar este artículo: Valencia-Méndez, D. L. (2022). Textos escritos en español como segunda lengua por estudiantes Sordos bilingües: un análisis desde la lingüística sistémico-funcional. Íkala, Revista de Lenguaje y Cultura, 27(1), 13-30. https://doi.org/10.17533/udea.ikala.v27n1a01 